\title{
ARTíCULO \\ Gasterópodos con potencial económico asociados al intermareal rocoso de la Región Marina Prioritaria 32, Guerrero, México
}

Gastropods with economic potential associated with the rocky intertidal zone of the Marine Region Priority 32, Mexico

\section{Carmina Torreblanca-Ramírez ${ }^{1}$, Rafael Flores-Garza², Pedro Flores-Rodríguez², Sergio García-Ibáñez ${ }^{2}$, Jesús Emilio Michel-Morfin ${ }^{3}$ y José Luis Rosas-Acevedo ${ }^{1}$}

${ }^{1}$ Unidad de Ciencias de Desarrollo Regional, Universidad Autónoma de Guerrero, Calle Pino s/n Colonia El Roble, C.P. 39640 Acapulco, Guerrero, México. carminatorreblanca@yahoo.com.mx

${ }^{2}$ Unidad Académica de Ecología Marina, Universidad Autónoma de Guerrero, Av. Gran Vía Tropical No. 20, Fraccionamiento Las Playas, C.P. 39390, Acapulco Guerrero, México. rfloresgarza@yahoo.com

${ }^{3}$ Universidad de Guadalajara, Departamento de Estudios para el Desarrollo Sustentable de Zonas Costeras, Av. Gómez Farías No. 82, San Patricio-Melaque Jalisco, C.P. 48980, México

\begin{abstract}
There are numerous species of gastropods of commercial importance; they are used for meat and for their shell, in addition to the potential use of their toxins for pharmaceutical use. There are few studies focused on the analysis of the importance and/or the economic potential of these organisms in the State of Guerrero, Mexico. This research was conducted at 7 sites, with the aim of determine the species of economic potential, the current use and possible commercial use, besides estimating the descriptive statistics of population sizes and geographic distribution. According to the regionalization of the National Commission for the Knowledge and Use of Biodiversity, the sampling sites are located in the Marine Region Priority 32. Species with economic potential were determined as a basis of the abundance of populations and the frequency of occurrence at the sampling sites. Forty species of economic potential were found, of which 14 are commercially exploited, one has biomedical potential and 25 of them no record was found indicating any commercial utilization. The species of greater size was Macrocypraea cervinetta. Sixty five percent of the species with economic potential are of wide distribution. The region is highly diverse and with potential for commercial use of a large number of species. To carry out a good fishery management of the species with economic potential, fishery-biology and ecological studies are required that allow for the development of measures and strategies for carrying out sound and sustainable use of these resources.
\end{abstract}

Key words: Gastropoda, species richness, utilization, size, Guerrero

Resumen.- Los gasterópodos tienen numerosas especies de importancia comercial, de ellos se utilizan la carne y concha, además del uso potencial de sus toxinas para uso farmacéutico. Son pocos los estudios enfocados al análisis de la importancia y/o el potencial económico de estos organismos en el Estado de Guerrero, México. Esta investigación se realizó en 7 sitios, con el objetivo de determinar las especies con potencial económico, el aprovechamiento actual y el posible uso comercial, estimar los estadísticos descriptivos de talla de las poblaciones y su distribución geográfica. De acuerdo a la regionalización de la Comisión Nacional para el Uso y Conocimiento de la Biodiversidad, los sitios pertenecen a la Región Marina Prioritaria 32. Las especies con potencial económico se determinaron teniendo como base la abundancia de sus poblaciones y la frecuencia de aparición en los sitios de muestreo. Se registraron 40 especies con potencial económico, de las cuales 14 ya son explotadas comercialmente, una tiene potencial biomédico y de 25 no se encontró registro de que sean explotadas de manera comercial. La especie de mayor talla fue Macrocypraea cervinetta. El $65 \%$ de las especies con potencial económico son de amplia distribución. La región es altamente diversa y con potencial para el uso comercial de un gran número de especies. Para llevar a cabo una buena administración pesquera de las especies con potencial económico, se requiere de estudios biológico pesqueros y ecológicos que permitan el desarrollo de medidas y estrategias para llevar a cabo un uso racional y sustentable de estos recursos.

Palabras clave: Gastropoda, riqueza, aprovechamiento, tallas, Guerrero 


\section{INTRODUCCIÓN}

En México, han sido delimitadas y caracterizadas por la Comisión Nacional para la Conservación y Uso de la Biodiversidad (CONABIO), 70 áreas costeras y oceánicas consideradas prioritarias por su alta diversidad biológica, por el uso de sus recursos y por su falta de conocimiento sobre su biodiversidad (Arriaga et al. 1998). En la costa del estado de Guerrero, se encuentran ubicadas 4 regiones marinas prioritarias sobre las cuales se ha señalado la falta de conocimiento de las especies marinas que habitan en estas regiones.

La Región Marina Prioritaria 32 (RMP 32), conocida como Coyuca-Tres Palos se encuentra ubicada en el Estado de Guerrero, tiene una extensión de $829 \mathrm{~km}^{2}$ y está clasificada como un área con alta biodiversidad, la cual se encuentra amenazada. La problemática que de acuerdo a la CONABIO presenta, es la modificación de su entorno por descargas de agua dulce, agroquímicos, fertilizantes y desechos ganaderos; daño al ambiente por el transporte turístico; usos de recursos naturales tales como especies de aves en riesgo; introducción de especies exóticas (como la tilapia y palma cocotera); así como el desconocimiento de la normatividad vigente para el aprovechamiento de los recursos naturales por otra parte de los usuarios del área (Arriaga et al. 1998).

El intermareal rocoso es uno de los hábitats que encontramos en la RMP 32 y es considerado como uno de los más diversos del ambiente marino (Tait \& Dipper 1998). Dicho hábitat se ubica en la costa que corresponde al municipio de Acapulco, su extensión es amplia y se caracteriza por presentar diversas condiciones microambientales, lo que genera espacios para que habiten gran variedad de especies, entre las que encontramos a los moluscos. Con respecto a este taxón, la CONABIO ha señalado que es un grupo sobre el cual se tiene poca información y que requiere de mayor investigación en toda la República Mexicana (Arriaga et al. 1998).

Los moluscos de la clase Gastropoda, llamados también univalvos o criaturas de una sola concha, son numerosos y conocidos del ambiente marino, presentan una gran diversidad de forma patrones, colores y esculturas de su concha, ya que puede ser convexa, plana, turriculada, cónica, hay ejemplares que no presentan concha o la tiene interna, cuya característica principal es su concha que se encuentra en forma de espiral, aún cuando no todos la presentan cuando son adultos, sino en alguna etapa de desarrollo (López \& Urcuyo 2009).
Los gasterópodos incluyen un gran número de especies de importancia comercial, su carne es usada como alimento y sus conchas como piezas de ornato, joyería y artesanías, además son utilizadas como fuente de productos cosméticos y farmacéuticos (Ríos-Jara et al. 2008).

Alcolado (2001), reconoce la utilidad de los estudios de la diversidad de moluscos y de sus asociaciones biológicas como indicadoras del grado de tensión y constancia ambiental, tanto en el medio marino como en el terrestre. Cuanto más se corresponden los valores de las variables ambientales con los intervalos fisiológicos favorables para la vida, mayor es el número de especies potenciales que pueden concurrir en un hábitat determinado y cuanto menos frecuentes y más pequeñas sean las fluctuaciones de esas variables, mayores posibilidades existen para que cada una de las especies exploten al máximo los recursos disponibles y aumenten sus poblaciones. Sin embargo, la riqueza de especies de moluscos o la heterogeneidad reflejan de forma comparativa el grado de estrés, la equidad y el nivel de pronóstico ambiental. Por lo tanto, el ecólogo se basa en la identidad de la especie o su dominancia (especies generalistas, de amplia distribución, con poblaciones abundantes, de tallas más grandes y que soportan más las variaciones ambientales), y en ocasiones en el censo de las especies ausentes (especies especialistas, más sensibles, sus poblaciones son más pequeñas, tiene menos resistencias a las variaciones ambientales). Así, si domina una especie resistente a la sedimentación, ese es el factor principal que debe estar actuando.

A lo largo del tiempo, los gasterópodos se han convertido en una fuente de alimentación y de recursos de importancia económica. Como todo recurso natural, debido a una administración y utilización inadecuada, corre el riesgo de agotarse. Para evitar que esto ocurra, se debe contar con estudios que evalúen la biodiversidad, las poblaciones y la comunidad, mismos que, permitan un uso adecuado y sostenible de estos recursos, así como, la recuperación de las especies y de su hábitat (Ríos-Jara et al. 2008).

Para una adecuada administración pesquera de moluscos en México, se requiere de la evaluación de especies por localidad, con conocimiento de la dinámica de sus poblaciones y ciclos reproductivos, que permitan determinar volúmenes de captura, tallas mínimas y períodos de explotación (Baqueiro \& Aldana 2003). 
La costa de la RMP 32, cuenta con una amplia extensión de litoral que, además de ofrecer al turismo hermosas playas, proporcionan una variedad de moluscos marinos comestibles de importancia gastronómica y una fuente importante de recursos económicos para los pescadores y servidores turísticos. De ahí que los moluscos marinos tengan un importante papel en la vida económica y cultural del municipio de Acapulco.

Para la República Mexicana existen algunos trabajos importantes sobre moluscos de importancia comercial como los de Baqueiro et al. (1982), Baqueiro \& Aldana (2003) y Ríos-Jara et al. (2008).

Sin embargo, la producción pesquera de moluscos de importancia comercial para el Estado de Guerrero ha sido poco estudiada (Villegas-Maldonado et al. 2007, FloresGarza et al. 2012) y los trabajos sobre moluscos para el Estado, son estudios básicamente de diversidad, estructura de la comunidad, distribución geográfica, distribución por zonas en el hábitat y como fauna asociada (Villalpando 1986, Valdés-González et al. 2004, Flores Rodríguez et al. 2007, Flores-Garza et al. 2011, Torreblanca-Ramírez et al. 2012).

El presente trabajo se desarrolló en la zona intermareal rocosa de la RMP 32 y tuvo como objetivos: 1) determinar las especies con potencial económico, 2) determinar el aprovechamiento actual y el posible uso comercial de las especies, 3) estimar los estadísticos descriptivos de las tallas en largo de las poblaciones con potencial económico y 4) analizar la distribución geográfica de las especies.

\section{Materiales y MÉTODOS}

La RMP 32, se encuentra ubicada entre $16^{\circ} 35^{\prime} 24^{\prime \prime}$ $17^{\circ} 28^{\prime} 12^{\prime \prime}$ E y $99^{\circ} 25^{\prime} 12^{\prime \prime}-100^{\circ} 33^{\prime} \mathrm{O}$ (Fig. 1). Presenta un clima cálido sub-húmedo, la temperatura media anual es mayor de $26^{\circ} \mathrm{C}$, en la región ocurren tormentas tropicales y huracanes de mayo a noviembre. Pertenece a la placa de Norteamérica, formada por rocas metamórficas y una plataforma estrecha. Está influida por la corriente costanera de Costa Rica y la corriente Norecuatorial, es una zona de oleaje alto y que recibe aporte de agua dulce por río. Se presentan procesos de turbulencia y eventos de marea roja y El Niño (Arriaga et al. 1998).

El muestreo se llevó en 7 sitios, durante el periodo de enero de 2009 a enero 2012, cada sitio se georeferenció y se describió de acuerdo a los siguientes criterios: longitud aproximada de la zona de muestreo, estructura del sustrato, tipo de roca y exposición al oleaje. Las descripciones de los sitios de colecta se realizaron con base en Mottana et al. (1980), en las cartas geológicas del Instituto Nacional de Estadística, Geografía e Informática (Acapulco E14-11, 1:50,000) y observaciones realizadas en campo.

Los sitios fueron: 1) Majahua (16 $47^{\prime} 40,49^{\prime \prime} \mathrm{N}$ y 9950’30, 29'O), la cual es una playa de acumulación o de aluvión, caracterizada por cantos rodados en procesos de abrasión, cuenta con bloques de textura lisa al tacto y poca presencia de fisuras y/o hendiduras y donde la intensidad del oleaje es baja; 2) Parque de la Reina (16 $51^{\prime} 02,27^{\prime \prime} \mathrm{N}$ y $99^{\circ} 54^{\prime} 01,70^{\prime}$ 'O), es una playa de acumulación artificial, de rocas de tamaño variable y consistencia porosa, presenta escombros, restos de piso, columnas de concreto y la intensidad del oleaje es medio; 3) Muelle (16 $50 ' 56,86^{\prime \prime} \mathrm{N}$ y 99 $54^{\circ} 02,39$ 'O $)$, playa de acumulación artificial, presenta rocas metamórficas del cretácico inferior Ki (metavolcánico) de tamaño variables y consistencia porosa, así como por material de desechos de construcción, la intensidad del oleaje es medio; 4) Tlacopanocha (16 50 '41,53'”N y 9954'25,02'O) y 5) Manzanillo (16 50 '27,90’'N y 9954'38,14’O), las cuales son playas de acumulación compuestas por rocas de tipo granito-granodiorita y por rocas metamórficas del cretácico inferior $\mathrm{Ki}$, que varían mucho en cuanto a las dimensiones, ya que es posible encontrar desde grava hasta grandes bloques, la superficie del sustrato es áspera al tacto, también es posibles apreciar material de desechos de construcción, como el concreto y por la ubicación de ambas playas la intensidad del oleaje es bajo; 6) La Angosta ( $16^{\circ} 60^{\prime} 29,86^{\prime \prime} \mathrm{N}$ y 9954'55,70'’O), está compuesta por rocas metamórficas del cretácico inferior Ki de tamaño variable de textura semi-áspera al tacto, y presencia de fisuras, hendiduras y oquedades, las intensidad del oleaje es alto (expuesta) y finalmente, 7) Pie de la Cuesta (1652’25,64’'N y 9956’34,64”O), es una playa de alto oleaje (expuestas), compuestas por rocas del cretácico inferior Ki. Se caracteriza por ser de macizos rocosos, el sustrato es áspero y fijo con presencia de fisuras o hendiduras y oquedades.

Se llevaron a cabo 3 muestreos en cada sitio. Todos los muestreos se realizaron, durante las horas de marea baja y los días de luna nueva. Para llevar a cabo la cuantificación de los organismos, se muestreó un área de $10 \mathrm{~m}^{2}$. El muestreo fue sistemático, el punto de partida del muestreo fue seleccionado al azar y luego una cuerda de longitud de $30 \mathrm{~m}$ se colocó en paralelo a la costa en la zona intermareal. Para delimitar la unidad de muestreo se utilizó un marco, que fue fabricado con tubos de PVC de 

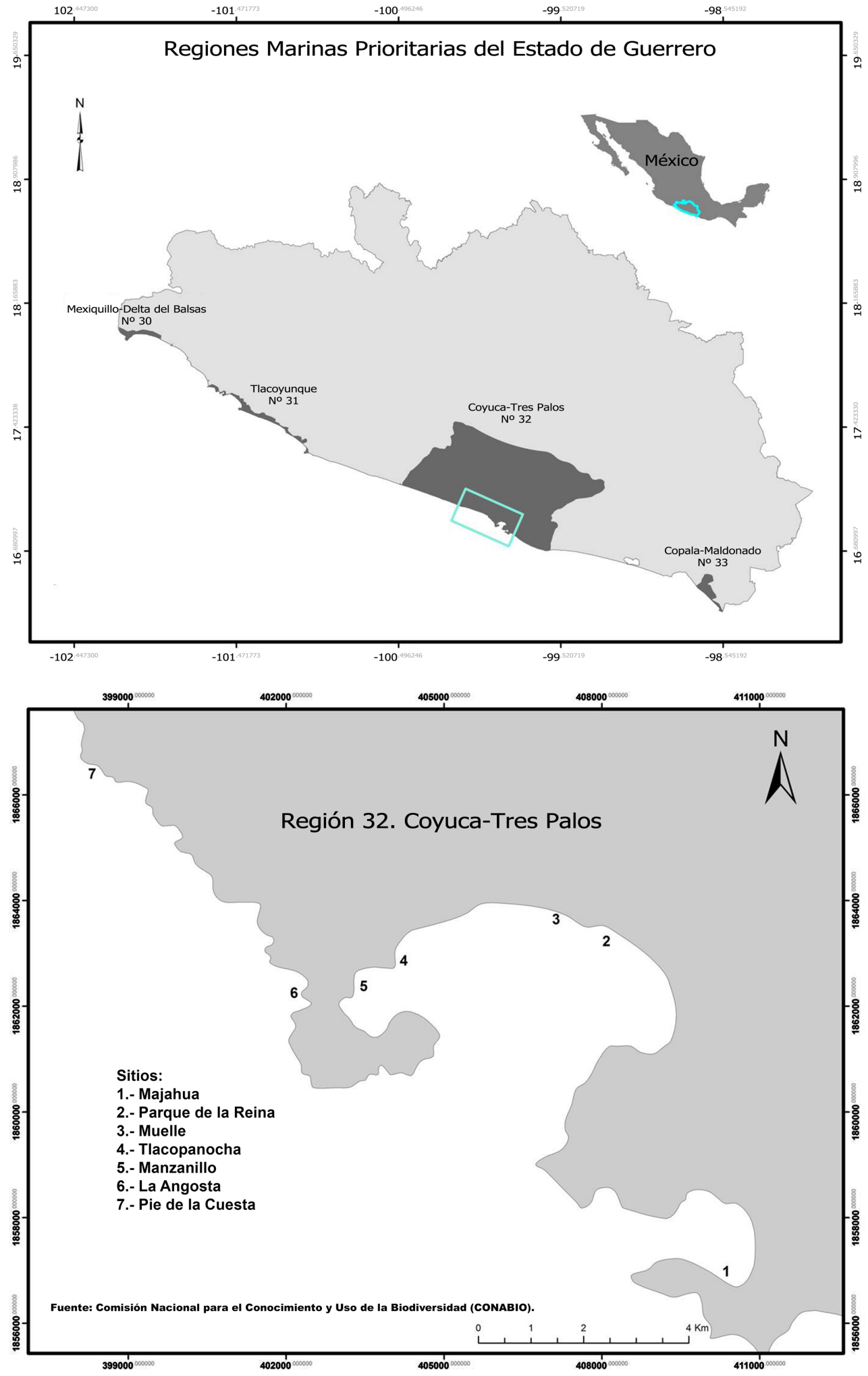

Figura 1. Regiones Marinas Prioritarias en el Estado de Guerrero, México y sitios de muestreo / Marine Priority Regions in the State of Guerrero, Mexico and sampling sites 
$1 \mathrm{x} 1 \mathrm{~m}$. Una vez que el punto de partida fue seleccionado, el marco que delimita la unidad de muestreo se colocó en el punto seleccionado. Todos los especímenes de Gastropoda vivos dentro de la unidad de muestreo fueron colocados dentro de un recipiente de plástico que contenía agua de mar y se protegió de la luz solar. Después de concluir la recolecta de la primera unidad de muestreo, se dejó un espacio de $2 \mathrm{~m}$ siguiendo la cuerda y se colocó el marco para delimitar el siguiente $\mathrm{m}^{2}$, donde se recolectaría la muestra. Este procedimiento se repitió hasta completar $10 \mathrm{~m}^{2}$. Se preservó la muestra colocando a los ejemplares en frascos con alcohol etílico al $96 \%$ y posteriormente trasladados al laboratorio. Los especímenes encontrados dentro de las unidades de muestreo fueron identificados y cuantificados. La adecuada ubicación taxonómica requirió de una fase de confirmación en el laboratorio, mediante el uso de literatura especializada (Keen 1971), la nomenclatura se actualizó de acuerdo a Skoglund (2002) y WoRMS (2013) ${ }^{1}$. Los ejemplares colectados fueron medidos en longitud $(\mathrm{mm})$. Posterior a la identificación y medición de los organismos, estos se depositaron en la Colección Malacológica De la Unidad Académica de Ecología Marina, Universidad Autónoma de Guerrero y en la Colección Nacional de Moluscos en la Universidad Nacional Autónoma de México.

\section{AnÁlisis de DATOS}

La riqueza se consideró como el número de especies de moluscos presentes. Las especies con potencial económico se determinaron teniendo como base la abundancia de sus poblaciones y su frecuencia de aparición en los sitios, para lo cual se utilizó el método gráfico de correlación de Olmstead-Tukey (Sokal \& Rohlf 1969), representado mediante gráficas de cuadrantes, que tuvo como base el cálculo de 2 estimadores, el primero fue el promedio de la abundancia relativa de todas las especies, expresado en logaritmo de la abundancia +1 y el segundo estimador, fue el promedio del número de muestras en la cual todas las especies están presentes (frecuencia de ocurrencia), expresado en porcentaje. Con el cálculo de estos estimadores, se analizó gráficamente el porcentaje de la frecuencia de aparición de cada especie, contra el porcentaje de la abundancia relativa promedio de cada especie, expresada como el logaritmo de la abundancia +1 . Las especies dominantes fueron aquellas cuyos valores de abundancia y frecuencia de ocurrencia sobrepasan la media aritmética de ambos valores estimados, estos valores indican que las especies que se clasifiquen en esta categoría son aquellas que soportan un mayor estrés ambiental y están mejor adaptadas a las diferentes condiciones que presenta el intermareal rocoso de la RMP 32, por lo que es posible que soporten con mayor facilidad el manejo que se requiere para que sean utilizadas como un producto comercial. La estimación de los estadísticos descriptivos en talla de las poblaciones que componen la comunidad de moluscos se realizó con base en el largo de la concha, obteniendo los valores máximos, mínimos, promedios y desviación estándar y se expresaron en milímetros $(\mathrm{mm})$.

Para analizar la distribución de las especies de gasterópodos en la RMP 32, se utilizó como criterio el porcentaje de sitios en la cual una especie estuvo presente (frecuencia de aparición) y se clasificó de la siguiente manera; a) amplia distribución: cuando la especie se presentó en por lo menos el $80 \%$ de los sitios y b) distribución regular: cuando la especie se colectó entre el 60 al $79 \%$ de los sitios.

\section{Resultados}

Se analizaron un total de 11,253 organismos, correspondientes a 28 familias, 65 géneros y 107 especies. El análisis gráfico de correlación arrojó que 40 especies de moluscos fueron dominantes, (Fig. 2). Entre estas se encuentra Conux nux (Broderip 1833), dicha especie se encuentra sujeta investigación, dado que es productora de toxinas con potencial biomédico y 14 especies ya son explotadas comercialmente, de las cuales, 8 especies son utilizadas como alimento para consumo humano: Crucibulum (Crucibulum) umbrella (Deshayes, 1830), Crucibulum (C.) scutellatum (Wood, 1928), Mancinella speciosa (Valenciennes, 1832), Mancinella triangularis (Blainville, 1832), Plicopurpura pansa (Gould, 1853), Stramonita biserialis (Blainville, 1832), Leucozonia cerata (Wood, 1828) y Opeatostoma pseudodon (Burrow, 1815), ya sea para venta a restaurantes o para autoconsumo; 8 tienen uso artesanal y ornamental (Lottia pediculus (Philippi, 1846), Lottia mesoleuca (Menke, 1851), Tectura fascicularis (Menke, 1851), Macrocypraea cervinetta (Kiener, 1843), Mauritia arabicula Lamarck, 1810. Jenneria pustulata [Lightfoot, 1786], L. cerata y O. pseudodon) y con ellas se elaboran elementos decorativos de uso personal, principalmente joyería, también son utilizadas

${ }^{1}$ WorRMS 2013. World Register of Marine Species. <http://www. marinespecies.org/aphia.php?p=taxdetails\&id=456599> 


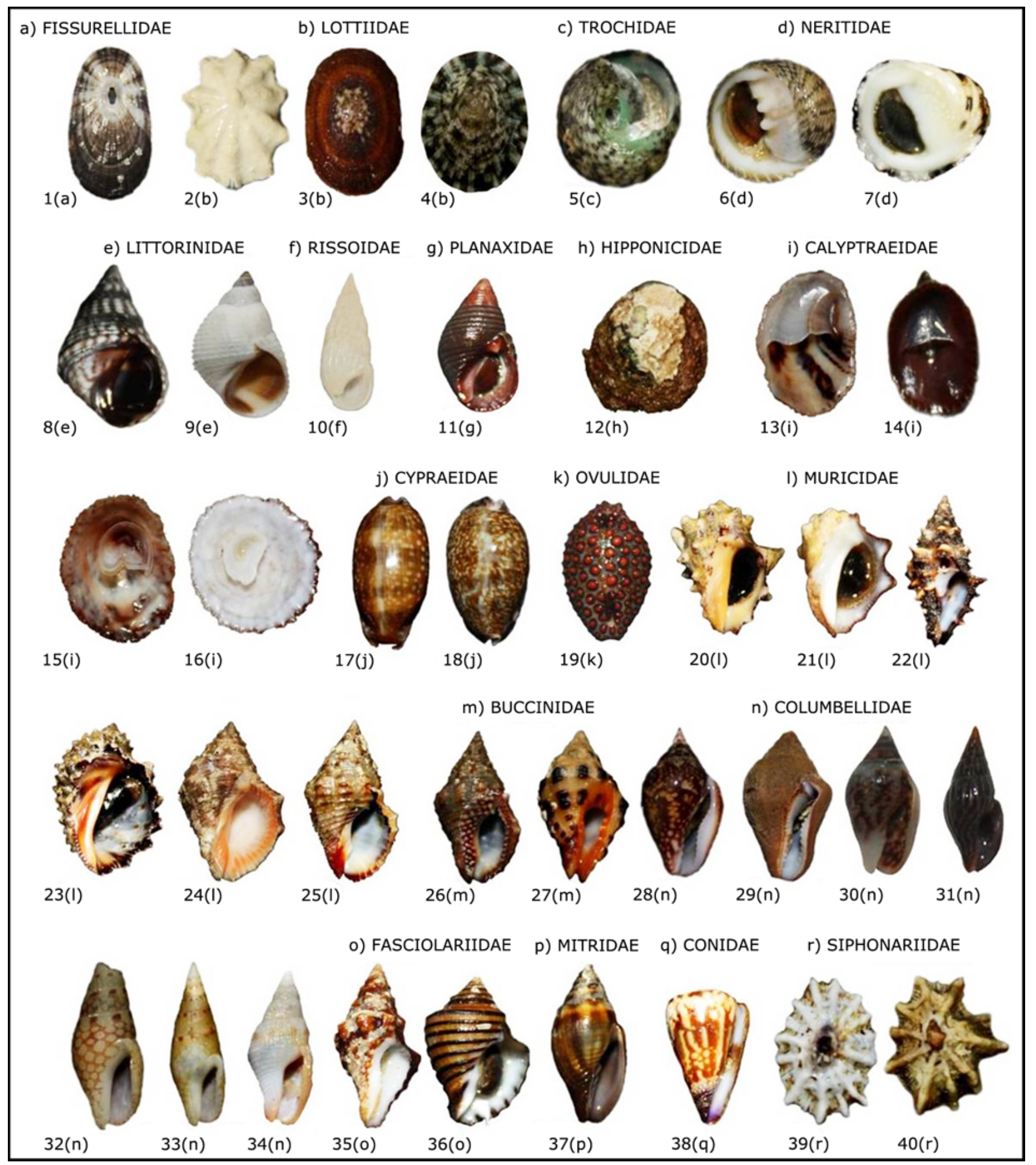

Figura 2. Especies de la clase Gastropoda con potencial económico de la región marina prioritaria 32, Guerrero, México. 1(a) Diodora inaequalis, 2(b) Lottia pediculus, 3(b) L. mesoleuca, 4(b) Tectura fascicularis, 5(c) Tegula (A.) globulus, 6(d) Nerita (C.) scabricosta, 7(d) N. (T.) funiculata, 8(e) Nodilittorina (N.) aspera, 9(e) N. (F.) modesta, 10(f) Rissoina (R.) stricta, 11(g) Planaxis obsoletus, 12(h) Hipponix delicatus, 13(i) Crepidula aculeata, 14(i) C. incurva, 15(i) Crucibulum (C.) scutellatum, 16(i) C. (C.) umbrella, 17(j) M acrocypraea cervinetta, 18(j) Mauritia arabicula, 19(k) Jenneria pustulata, 20(I) Mancinella speciosa, 21(I) M. triangularis, 22(I) M uricopsis (M.) zeteki, 23(I) Plicopurpura pansa, 24(I) Stramonita 
biserialis, 25(I) Trachypollia lugubris, $26(\mathrm{~m})$ Cantharus (P.) sanguinolentus, 27(m) Engina tabogaensis, 28(n) Columbella fuscata, 29(n) C. major, 30(n) C. sonsonatensis, 31(n) Costoanachis nigrofusca, 32(n) Mitrella ocellata, 33(n) M. xenia, 34(n) Cosmioconcha palmeri, 35(0) Leucozonia cerata, 36(o) Opeatostoma pseudodon, 37(p) Mitra (S.) tristis, 38(q) Conux nux, 39(r) Siphonaria (H.) maura y 40(r) S. (H.) palmata. Fotos colección de invertebrados marinos, Unidad Académica de Ecología Marina, Universidad Autónoma de Guerrero. Autores: Carmina Torreblanca-Ramírez y Rafael Flores-Garza / Class Gastropoda with economic potential in the marine priority region 32, Guerrero, Mexico. 1(a) Diodora inaequalis, 2(b) Lottia pediculus, 3(b) L. mesoleuca, 4(b) Tectura fascicularis, 5(c) Tegula (A.) globulus, 6(d) Nerita (C.) scabricosta, 7(d) N. (T.) funiculata, 8(e) Nodilittorina (N.) aspera, 9(e) N. (F.) modesta, 10(f) Rissoina (R.) stricta, 11(g) Planaxis obsoletus, 12(h) Hipponix delicatus, 13(i) Crepidula aculeata, 14(i) C. incurva, 15(i) Crucibulum (C.) scutellatum, 16(i) C. (C.) umbrella, 17(j) Macrocypraea cervinetta, 18(j) M auritia arabicula, 19(k) Jenneria pustulata, 20(I) M ancinella speciosa, 21(I) M. triangularis, 22(I) M uricopsis (M.) zeteki, 23(I) Plicopurpura pansa, 24(I) Stramonita biserialis, 25(I) Trachypollia lugubris, 26(m) Cantharus (P.) sanguinolentus, $27(\mathrm{~m})$ Engina tabogaensis, 28(n) Columbella fuscata, 29(n) C. major, 30(n) C. sonsonatensis, 31(n) Costoanachis nigrofusca, 32(n) Mitrella ocellata, 33(n) M. xenia, 34(n) Cosmioconcha palmeri, 35(o) Leucozonia cerata, 36(o) Opeatostoma pseudodon, 37(p) Mitra (S.) tristis, 38(q) Conux nux, 39(r) Siphonaria (H.) maura and 40(r) S. (H.) palmata. Pictures collection of marine invertebrates, Unidad Académica de Ecología Marina, Universidad Autónoma de Guerrero. Authors: Carmina Torreblanca-Ramírez and Rafael Flores-Garza

como objetos decorativos o de uso común como cortinas, cuadros, espejos; además una especie, el caracol $P$. pansa también es utilizado para teñir telas y se caracteriza por la secreción de un líquido que desde las época prehispánica se ha explotado a través de la ordeña de los organismos sin sacrificarlos. De 25 especies determinadas con potencial económico no se encontraron registros de que fueran utilizadas comercialmente en la región (Tabla 1).

En relación a la composición de tallas de las poblaciones con potencial económico, M. cervinetta fue la especie que registró la mayor talla con $84,48 \mathrm{~mm}$ (mínimo=4,51; 35,76 $\pm 24,61$ ), seguida por Crucibulum (Crucibulum) umbrella (Deshayes, 1830) con 57,3 mm (mínimo=8,39; 35,51 $\pm 12,36$ ). La de menor talla la presentó Nodilittorina (Fossarilittorina) modesta (Philippi, 1844) con $1.5 \mathrm{~mm}$ (máximo= 35,1; 7,31 $\pm 3,84$ ), seguida por Nodilittorina (Nodilittorina) aspera (Philippi, 1846) con 1,9 mm (máximo=15,4; 5,88 \pm 3,77), Siphonaria (Heterosiphonaria) palmata Carpenter, 1857 con 1,14 mm (máximo=16,08; 7,3 $\pm 2,87$ ) y Siphonaria (H.) maura Sowerby, 1835 con 1,33 mm (máximo=19, 52; 8,13 $\pm 3,25$ ) (Tabla 1).

En cuanto a la distribución geográfica, se determinó que el $65 \%$ de las especies determinadas con potencial económico se encuentran presentes en al menos 6 sitios (Tabla 2).

\section{Discusión}

La riqueza de especies con potencial económico que se reporta para la RMP 32 es alta comparada con lo reportado por Ríos-Jara et al. (2008), ya que ellos reportan 16 especies de Gasterópodos de importancia comercial y potenciales para las costas de Oaxaca y Chiapas.

En este estudio, se encontraron 15 especies que ya son explotadas comercialmente y son aprovechadas principalmente como alimento, para elaborar artesanías o para teñir prendas ceremoniales o bien especies que están en investigación para uso biomédico. A pesar del uso que se tiene de estas especies, no existen normas o lineamientos para su explotación o bien para la protección o manejo de la zona intermareal. Las excepciones las constituye en $P$. pansa y $C$. scutellatum que aparecen bajo protección en la (NOM-059-SEMARNAT-2010)2 ${ }^{2}$. Aunque las abundancias y frecuencias de aparición de las especies con potencial económico son altas, lo que indica la capacidad de estas especies para soportar el estrés ambiental y la presión por pesca, es importante reglamentar lo más rápido posible el manejo de estos recursos antes que daños irreparables afecten las poblaciones.

Las otras 25 especies restantes, no han sido explotadas comercialmente en la región, pero debido a las características físicas de la concha, la diversidad de formas, colores, tallas pequeñas, se consideran con un potencial uso ornamental y/o artesanal.

${ }^{2}$ Norma Oficial Mexicana NOM-059-SEMARNAT-2010. Protección ambiental-especies nativas de México de flora y fauna silvestres-categorías de riesgo y especificaciones para su inclusión, exclusión o cambio - lista de especies en riesgo. Diario Oficial de la Federación. 30 de diciembre de 2010. <http://www.semarnat.gob.mx/temas/gestionambiental/vidasilvestre/ Documents/NOM_059_SEMARNAT_2010.pdf> 
Tabla 1. Lista de especies y familias de la Clase Gastropoda con potencial económico asociada al sustrato intermareal rocoso en la región marina prioritaria 32, Guerrero, México. Tallas en largo y ancho / List of species and families of the Class Gastropoda with economic potential associated with intertidal rocky substrate in the marine region priority 32 , Guerrero Mexico. Sizes in length and width

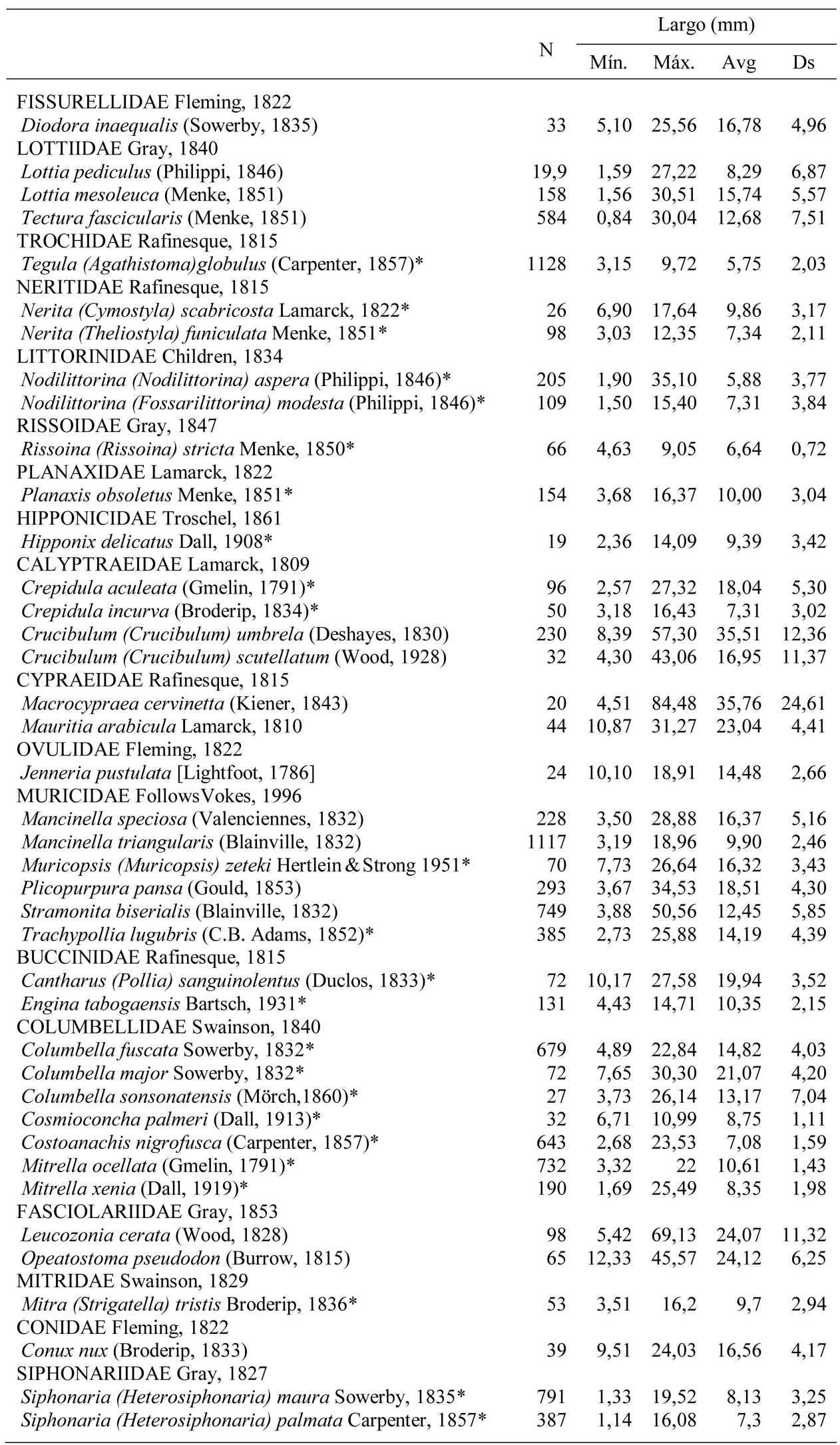


Tabla 2. Distribución y abundancias por sitios de muestreo de las especies de la Clase Gastropoda con potencial económico y asociadas con el intermareal rocoso en la región marina prioritaria 32, Guerrero, México. Sitios de muestreos: 1. Majahua, 2. Parque de la Reina, 3. Muelle, 4. Tlacopanocha, 5. Manzanillo, 6. La Angosta y 7. Pie de la Cuesta / Distribution and abundance by sampling sites of the species of the Class Gastropoda with economic potential and are associated with rocky intertidal in the marine priority region 32, Guerrero, Mexico. Sampling sites: 1. Majahua, 2. Parque de la Reina, 3. Muelle, 4. Tlacopanocha, 5. Manzanillo, 6. La Angosta and 7. Pie de la Cuesta

\begin{tabular}{|c|c|c|c|c|c|c|c|c|}
\hline & 1 & 2 & 3 & 4 & 5 & 6 & 7 & Distribución \\
\hline Diodora inaequalis & 5 & 4 & & 3 & 20 & 1 & & Regular \\
\hline Lottia pediculus & 2 & 8 & 38 & 37 & 4 & 110 & & Amplia \\
\hline Lottia mesoleuca & 87 & 16 & 3 & 5 & 1 & 3 & 43 & Amplia \\
\hline Tectura fascicularis & 34 & 51 & 276 & 98 & 22 & 54 & 49 & Amplia \\
\hline Tegula (A.) globulus & 64 & 199 & 843 & & 18 & & 4 & Regular \\
\hline Nerita (C.) scabricosta & 2 & 13 & 1 & & 6 & & 4 & Regular \\
\hline Nerita (T.) funiculata & 49 & 11 & 1 & & 36 & & 1 & Regular \\
\hline Nodilittorina (N.) aspera & 65 & 70 & 58 & & 9 & & 3 & Regular \\
\hline Nodilittorina (F.) modesta & 36 & 15 & & 8 & & 4 & 46 & Regular \\
\hline Rissoina (R.) stricta & 1 & 2 & 52 & 9 & 1 & 1 & & Amplia \\
\hline Planaxis obsoletus & 59 & 1 & 19 & 11 & & 5 & 59 & Amplia \\
\hline Hipponix delicatus & 14 & 1 & & 3 & 1 & & & Regular \\
\hline Crepidula aculeata & 30 & 4 & 1 & 2 & 59 & & & Regular \\
\hline Crepidula incurva & 19 & 21 & 2 & & 8 & & & Regular \\
\hline Crucibulum (C.) umbrella & 142 & 53 & 13 & 8 & 10 & 3 & 1 & Amplia \\
\hline Crucibulum (C.) scutellatum & 17 & 2 & 1 & 2 & 4 & 3 & 3 & Amplia \\
\hline Macrocypraea cervinetta & 2 & 7 & 7 & 1 & 2 & 1 & & Amplia \\
\hline Mauritia arabicula & 7 & 14 & 8 & 4 & 11 & & & Regular \\
\hline Jenneria pustulata & 4 & 4 & 1 & & 15 & & & Regular \\
\hline Mancinella speciosa & 8 & 144 & 27 & 6 & 9 & 33 & 1 & Amplia \\
\hline Mancinella triangularis & 149 & 113 & 354 & 41 & 34 & 226 & 200 & Amplia \\
\hline Muricopsis (M.) zeteki & 6 & 4 & 30 & 1 & 28 & 1 & & Amplia \\
\hline Plicopurpura pansa & 3 & 1 & 2 & 36 & 3 & 133 & 115 & Amplia \\
\hline Stramonita biserialis & 85 & 227 & 179 & 57 & 81 & 38 & 82 & Amplia \\
\hline Trachypollia lugubris & 25 & 46 & 104 & 23 & 30 & 153 & 4 & Amplia \\
\hline Cantharus (P.) sanguinolentus & 11 & 24 & 7 & 4 & 17 & 9 & & Amplia \\
\hline Engina tabogaensis & 64 & 17 & 3 & 2 & 42 & 3 & & Amplia \\
\hline Columbella fuscata & 156 & 25 & 334 & 72 & 75 & 14 & 3 & Amplia \\
\hline Columbella major & 6 & 10 & 17 & 2 & 31 & 6 & & Amplia \\
\hline Columbella sonsonatensis & 7 & 7 & & 1 & 3 & 9 & & Regular \\
\hline Cosmioconcha palmeri & 16 & 6 & 7 & 1 & & 2 & & Regular \\
\hline Costoanachis nigrofusca & 61 & 59 & 376 & 80 & 63 & 1 & 3 & Amplia \\
\hline Mitrella ocellata & 32 & 61 & 177 & 47 & 72 & 216 & 127 & Amplia \\
\hline Mitrella xenia & 13 & 5 & 110 & 8 & 11 & & 43 & Amplia \\
\hline Leucozonia cerata & 15 & 26 & 5 & 4 & 30 & 11 & 7 & Amplia \\
\hline Opeatostoma pseudodon & 1 & 14 & 19 & 3 & 4 & 24 & & Amplia \\
\hline Mitra (S.) tristis & 8 & 11 & 3 & & 31 & & & Regular \\
\hline Conux nux & 5 & 4 & & 6 & 24 & & & Regular \\
\hline Siphonaria (H.) maura & 564 & 14 & 64 & 35 & 79 & 12 & 23 & Amplia \\
\hline Siphonaria (H.) palmata & 307 & 5 & 21 & 24 & 29 & & 1 & Amplia \\
\hline
\end{tabular}


Con relación a las especies encontradas en la presente investigación se coincide en 6 especies que reportan importancia comercial para el consumo humano con Flores-Garza et al. (2012); Muricanthus princeps (Broderip, 1833), reportado en el trabajo antes citado, es una especie que fue encontrada en el intermareal rocoso de la RMP 32, pero de acuerdo al análisis realizado no se considera con potencial económico en el intermareal rocoso, dicha especie tiene su hábitat preferencial en la zona submareal, por lo tanto, son mayores sus abundancias en dicha zona.

Se consideran que las especies encontradas en el presente estudio, debido a su abundancia relativa y su amplia distribución en la región, son especies representativas de la RMP 32. Los moluscos, como todo recurso natural, deben de tener un manejo adecuado con la finalidad de lograr su uso sustentable. Para una buena administración pesquera de las especies con potencial económico de la clase Gastropoda en la RMP 32, se requiere de estudios biológico-pesqueros y ecológicos por localidad, así como del conocimiento de la dinámica de poblaciones, que permita determinar volúmenes de captura, tallas máximas y mínimas, periodos de explotación y por lo tanto desarrollar medidas y estrategias que permitan el uso sustentable de estos recursos.

\section{Agradecimientos}

El primer autor agradece al Consejo de Ciencia y Tecnología, México (CONACYT), por el apoyo otorgado a través de una beca para realizar mis estudios de posgrado en el doctorado de Ciencias Ambientales, Unidad de Ciencias de Desarrollo Regional de la Universidad Autónoma de Guerrero, México, y a la Dra. María Laura Sampedro Rosas por su apoyo y gestión en la beca CONACYT.

\section{LITERATURA CITADA}

Alcolado MP. 2001. Diversidad y bioindicación ambiental en el mar. Instituto de Oceanología. <http://oceanologia. redciencia.cu/articulos/articulo08.pdf $>$

Arriaga L, E Vázquez, J González, R Jiménez, E Muñoz \& V Aguilar. 1998. Regiones prioritarias y planeación para la conservación de la biodiversidad, en Capital natural de México. Estado de conservación y tendencias de cambio, pp, 433-457. CONABIO, México.

Baqueiro E \& D Aldana. 2003. Patrones en la biología poblacional de moluscos de importancia comercial en México. Revista de Biología Tropical 51(4): 97-107.
Baqueiro E, J Massó \& H Guajardo. 1982. Distribución y abundancia de moluscos de importancia comercial en Baja California Sur, México. Instituto Nacional de la Pesca, México, Serie de Divulgación 11: 1-32.

Flores-Garza R, C Torreblanca-Ramírez, P FloresRodríguez, S García-Ibáñez, L Galeana-Rebolledo, A Valdés-González \& A Rojas-Herrera. 2011. Mollusc community from a rocky intertidal zone in Acapulco, Mexico. Biodiversity 12(3): 144-153.

Flores-Garza R, S García-Ibáñez, P Flores-Rodríguez, C Torreblanca-Ramírez, L Galeana-Rebolledo, A ValdésGonzález, A Suástegui-Zárate \& J Violante-González. 2012. Commercially important marine mollusks for human consumption in Acapulco, México. Natural Resources 3: 248-260.

Flores-Rodríguez P, R Flores-Garza, S García-Ibáñez \& A Valdés-González. 2007. Variación en la diversidad malacológica del mesolitoral rocoso en Playa Troncones La Unión, Guerrero, México. Revista Mexicana de Biodiversidad 78: 33S-40S.

Keen AM. 1971. Sea shells of Tropical West America, 1064 pp. Stanford University Press, California.

López FA \& JR Urcuyo. 2009. Moluscos de Nicaragua II, Gastrópodos, 181 pp. Marena-Araucaria, UCA, Managua.

Mottana A, R Crespi \& G Liborio. 1980. Guía de minerales y rocas, 605 pp. Editorial Grijalbo, Barcelona.

Ríos-Jara E, CM Navarro-Caravantes, S Sarmiento, CM Galván-Villa \& E López-Uriarte. 2008. Bivalvos y gasterópodos (Mollusca) de importancia comercial y potencial de las costas de Chiapas y Oaxaca, México. Revista Ciencia y Mar 12(35): 3-20.

Skoglund C. 2002. Panamic province molluscan literature additions and changes from 1971 through 2001, III Gastropoda, 286 pp. The Festivus, San Diego.

Sokal RR \& FJ Rohlf. 1969. Biometry. The principles and practices of statistics in biological research, $776 \mathrm{pp}$. W. H. Freeman, San Francisco.

Taitt RV \& FA Dipper. 1998. Elements of marine ecology, 462 pp. Butterworth-Heinemann, Oxford.

Torreblanca-Ramírez C, R Flores-Garza, P FloresRodríguez, S García-Ibáñez \& L Galeana-Rebolledo. 2012. Riqueza, composición y diversidad de la comunidad de moluscos asociada al sustrato rocoso intermareal de playa Parque de la Reina, Acapulco, México. Revista de Biología Marina y Oceanografía 47(2): 283-294. 
Valdés-González A, P Flores-Rodríguez, R Flores-Garza \& S García-Ibáñez. 2004. Molluscan communities of rocky intertidal zone at two sites with different wave action on Isla la Roqueta, Acapulco, Guerrero, México. Journal of Shellfish Research 23: 875-880.

Villalpando E. 1986. Diversidad y zonación de moluscos de superficie rocosa, Isla Roqueta, Acapulco, Guerrero. Tesis de Licenciatura, Facultad de Ciencias, Universidad Nacional Autónoma de México, México, 150 pp.
Villegas-Maldonado S, E Neri-García, R Flores-Garza, S García-Ibáñez, P Flores-Rodríguez \& DG AranaSalvador. 2007. Datos preliminares de la diversidad de moluscos para el consumo humano que se expenden en Acapulco, Guerrero. En: Ríos-Jara E, MC EsquedaGonzález \& CM Galván-Villa (eds). Estudios sobre la Malacología y Conquiliología en México, pp. 56-58. Universidad de Guadalajara, Guadalajara.

Recibido el 13 de enero de 2014 y aceptado el 24 de septiembre de 2014

Editor: Claudia Bustos D. 